\title{
Editorial
}

The Editorial Board of Earth, Planets and Space wishes to thank the following reviewers for their help in evaluating papers for the year 1999.

\section{List of Reviewers for 1999}

Adam, A.

Agarwal, A. K.

Anderson, J. G.

Araki, S.

Araki, T.

Arnadottir, T.

Aso, T.

Banks, R.

Barraclough, D.

Blix, T.-A.

Chang, J.

Chanin, M.-L.

Chapman, C. R.

Chauvel, C.

Chouet, B. A.

Cortini, M.

Curto, J. J.

Davis, D. R.

Dewan, E.

Draeger, U.

Dubois, J.

Durda, D.

Eckermann, S.

Egbert, G. D.

Eisel, M.

Elford, W. G.

Engels, M.

Espy, P.

Everett, M.

Ferguson, I.

Floberghagen, R.

Frisch, P.

Fujinawa, Y.

Fujita, S.

Fujiwara, A.

Fujiwara, S.

Fuller, M.

Furiwara, H.

Gardner, C. S.

Geller, M. A.

Gibson-Wilde, D.

Grard, R.

Hagan, M. E.

Hagstrum, J. T.

Hanawa, T.
Harinarayana, T.

Harmsen, S. C.

Harrison, C. G. A.

Haslinger, F.

Hayashi, K.

Hernandez, G.

Hill, R.

Hirata, N.

Hiroi, T.

Hirooka, T.

Hirorta, I.

Hisada, Y.

Hjelt, S.-E.

Hobbs, B.

Hocking, W. K.

Hoegy, W.

Hoffmann, P.

Honkura, Y.

Horinouchi, T.

Hoshi, H.

Hyodo, M.

Iidaka, T.

Imoto, $\mathrm{M}$.

Ingham, $\mathrm{M}$.

Ishikawa, N.

Ishikawa, Y.

Ishimoto, $\mathrm{H}$.

Ishiwatari, $\mathrm{M}$.

Iwagami, N.

Iwasaki, $\mathrm{T}$.

Iwata, T.

Iyemori, $\mathrm{T}$.

Jankowski, J.

Jiracek, G.

Johnson, R. E.

Johnston, M.

Joselyn, J. A.

Junge, A.

Kafkalidis, J.

Kanekal, S. G.

Kato, N.

Kikuchi, M.

Kikuchi, T.

Kodama, K.

Kogiso, T.
Koike, M.

Kono, M.

Korja, T.

Kristjansson, L.

Kuge, K.

Lee, $\mathrm{K}$.

Lemoine, F. G.

Leroy, M. M.

Lieberman, R.

Lilley, F. E. M.

Louarn, P.

Lübkeu, F.-J.

Maezawa, K.

Makino, M.

Mao, A

Marquis, G.

Marubashi, K.

Marzocchi, W.

Masuda, K.

Matsui, $\mathrm{H}$.

Matsushima, M.

Mauson, A. H.

McDonnell, J. A. M.

McKnight, J. D.

Mcpherron, R. L.

Meek, C. E.

Menvielle, M.

Meriwether, J. W.

Meyer, C.

Miura, A.

Miyoshi, Y.

Morioka, A.

Morris, A.

Mulargia, F.

Munekane, $\mathrm{H}$.

Murayama, Y.

Nagasawa, C.

Nakagawa, T.

Nakamura, A. M.

Nakamura, M.

Nakamura, T.

Nakanishi, I.

Newman, G.

Ngan, K.

Nishida, Y.
Nogami, K.

Oda, $\mathrm{H}$.

Ogawa, T.

Ogawa, Y.

Ohtsuki, K.

Okada, M.

Okada, T.

Onsager, T. G.

Osella, A.

Oshiman, N.

Oyama, K.

Padilha, A.

Pancheva, D.

Papitashvili, V.

Parkinson, M. L.

Paxton, L. J.

Pek, J.

Pirjola, R.

Polet, J.

Portnyagin, Y.

Purcaru, G.

Raiche, A.

Ritter, P.

Roy, M.

Röttger, J.

Ryabov, V. B.

Sagiya, T.

Saikia, C. K.

Saito, A.

Sakanoi, T.

Saltus, R.

Sasaki, Y.

Sato, K.

Satomura, T.

Schnegg, P.-A.

Schnepp, E.

Schott, J. J.

Seno, T.

Shaw, J.

Shimizu, H.

Shinagawa, $\mathrm{H}$.

Shinohara, I.

Sica, R. J.

Simpson, F.

Singer, B. Sh. 
Singer, W.

Slavin, J.

Spichak, V. V.

Stănică, M.

Stening, R.

Stewart, G.

Suda, N.

Sugiura, M.

Summers, D.

Szarka, L.

Taguchi, S.

Tajika, E.

Takada, T.

Takahashi, H.

Takahashi, M.

Takakura, S.

Takenaka, H.

Tanaka, H.
Tanaka, T.

Tanaka, Y.

Terasawa, $\mathrm{T}$.

Thorsen, D.

Thraue, E. V.

Tomeoka, K.

Torta, J. M.

Tsuda, $\mathrm{T}$.

Tsunomura, S.

Tsurutani, B. T.

Tsyganenko, N. A.

Uchida, T.

Utada, $\mathrm{H}$.

Uyeshima, M.

Vallianatos, F.

Vanyan, L.

Varentsov, I.

Varentsov, I. M.
Vennerstroem, S.

Vial, F.

Vincent, R. A.

Wang, D. Y.

Wang, J.-H.

Wannamaker, $\mathrm{P}$.

Ward, W.

Watanabe, $\mathrm{H}$.

Watanabe, N.

Watanabe, S.

Watanabe, T.

Webb, F.

Weidelt, $P$.

Weinberg, J. L.

Witt, G.

Wyss, M.

Yamamoto, M.

Yamamoto, T.
Yamawaka, M. D.

Yamazaki, K.

Yee, J. H.

Yeoman, T.

Yokokura, T.

Yokoyama, Y.

Yomogida, K.

Yoshikawa, A.

Yoshikawa, M.

Yu, V. A.

Yung, Y. L.

Zahnle, K. J.

Zhang, K.

Zhao, D.

Zhao, G.

Zhao, X.

Zlotnicki, J. 